Title: Evaluation of the Psychometric Properties of the Social Isolation Measure (SIM) in Adults with Hearing Loss

Authors: Eithne Heffernan ${ }^{\mathrm{a}, \mathrm{b}, *}$, Alia Habiba,b, ${ }^{\mathrm{a}, \mathrm{c}}$ Melanie Ferguson ${ }^{\mathrm{a}, \mathrm{b}, \mathrm{d}}$

${ }^{a}$ National Institute for Health Research (NIHR) Nottingham Biomedical Research

Centre, Ropewalk House, 113 The Ropewalk, Nottingham, UK

${ }^{\mathrm{b}}$ Hearing Sciences Section, Division of Clinical Neuroscience, School of Medicine, University of Nottingham, Nottingham, UK

${ }^{\mathrm{c}}$ School of Life \& Health Sciences, Aston University, Birmingham, UK

d Nottingham University Hospitals National Health Service (NHS) Trust, Queens Medical Centre, Nottingham, UK

*Corresponding author: Eithne Heffernan, NIHR Nottingham Biomedical Research Centre, Ropewalk House, 113 The Ropewalk, Nottingham, NG1 5DU, UK

\title{
Email: eithne.heffernan1@nottingham.ac.uk
}

\author{
Abbreviations: \\ BOS - Bristol Online Survey \\ BRC - Biomedical Research Centre \\ CBT - Cognitive Behavioural Therapy \\ HHIE - Hearing Handicap Inventory for the Elderly \\ NIHR - National Institute for Health Research \\ SIM - Social Isolation Measure \\ UCLA - University of California, Los Angeles \\ WHODAS2.0 - World Health Organization Disability Assessment Schedule 2.0
}




\begin{abstract}
This study aimed to evaluate the psychometric properties of a brief, hearing-specific outcome measure: the Social Isolation Measure (SIM). In Phase 1, adults with hearing loss were invited to complete an online survey that contained the SIM, a hearing-specific participation questionnaire, a generic activity and participation questionnaire, and a generic loneliness questionnaire. In Phase 2, the participants were asked to complete the SIM for a second time 2-3 weeks following Phase 1. One hundred and sixteen adults with hearing loss completed Phase 1. Ninety-five participants also completed Phase 2. Twenty-nine participants were excluded from the Phase 2 data analysis because they reported that their hearing had changed since Phase 1 or because they completed Phase 2 outside of the 2-3 week interval following Phase 1. In support of its construct validity, the SIM had a strong correlation with the hearing-specific questionnaire and moderate correlations with the generic questionnaires. The findings also supported the internal consistency, interpretability, and test-retest reliability of the SIM. In conclusion, the SIM was found to have strong psychometric properties. It could serve as a brief measure of perceived social isolation in research or clinical practice.
\end{abstract}




\section{Introduction}

Social isolation is one of the major consequences of hearing loss (Strawbridge et al. 2000; Vas et al. 2017). It has been defined as a state in which an individual lacks engagement with others, lacks a sense of social belonging, lacks fulfilling relationships, and has a limited number of social connections (Nicholson Jr. 2009). It can be conceptualized as consisting of an objective component (i.e. actual social isolation), which refers to having a minimal number of social contacts, and a subjective component (i.e. perceived social isolation), which refers to the emotional experience of lacking companionship, support, and participation in society (Hawthorne 2008). Previous research has demonstrated that hearing loss can lead to both actual and perceived social isolation. For example, studies have found that individuals with hearing loss can experience a reduction in the size of their social networks and in the frequency of their social interactions (Kramer et al. 2002; Mick and Pichora-Fuller 2016). In addition, individuals have reported experiencing feelings of isolation and loneliness, even when they are in the company of other people, as a consequence of their hearing loss (Heffernan et al. 2016; Vas et al. 2017). Social isolation is a growing concern because it has been related to a range of serious health conditions and health states, including depression, cognitive decline, heart disease, stroke, and mortality (Glass et al. 2006; Shankar et al. 2013; Steptoe et al. 2013; Valtorta et al. 2016).

One of the main aims of adult auditory rehabilitation is to improve social participation and thus reduce isolation in adults with hearing loss (Boothroyd 2007; Ferguson et al. 2017). In order to assess whether adult auditory rehabilitation successfully achieves this aim, it is necessary to have a valid, hearing-specific social isolation outcome measure. In particular, there is a need for a brief (i.e. short form), valid, hearing-specific social isolation outcome measure (NHS England 2015; 2016). The primary advantage of brief outcome measures is that they are 
less burdensome for patients, especially those who are frail or who do not have a high degree of literacy (Garland et al. 2003; Hawthorne 2006; Reeve et al. 2013). Additionally, completing lengthy inventories about negative subject matter could potentially lead to resistance or denial in patients (Hawthorne 2006). Brief measures are also beneficial for clinicians because the burden associated with administering and scoring lengthy or complex instruments is frequently cited as a barrier to outcome measurement in clinical practice (Dillon and So 2000; Garland et al. 2003; Duncan and Murray 2012). Furthermore, brief measures are useful in many research contexts, such as telephone surveys and studies in which core outcome sets are applied (Hughes et al. 2004; Gierveld and Tilburg 2006; Prinsen et al. 2016).

Various brief measures of social isolation have been developed and utilized in healthcare research, such as the 8-item Social Disconnectedness Scale (Cornwell and Waite 2009a) and the 6-item Friendship Scale (Hawthorne 2006). In hearing research, a variety of brief social isolation measures have been used, particularly brief actual (i.e. objective) social isolation measures. For example, in a study by Mick and Pichora-Fuller (2016), social isolation in adults with hearing loss was measured using items from the National Health and Nutrition Examination Survey Social Support Questionnaire (2005). These items asked whether the respondents are married or in a domestic partnership, have close friends, have financial support, and have emotional support. Keidser and Seeto (2017) measured social isolation in adults with hearing loss using two items that assessed frequency of visits from family or friends and frequency of engagement in social activities. In a study examining hearing and vision loss in older adults, Jang et al. (2003) utilised Lubben's (1988) Social Network Scale, which includes items that assess the number of relatives and friends seen regularly by the respondent and the number of relatives or friends that are close to the respondent. They also measured social support using items originally developed 
by Krause and Borawski-Clark (1995), which asked respondents how often they received instrumental support (e.g. help with chores), informational support (e.g. sharing suggestions and information), and emotional support (e.g. having others listen to them). Respondents also reported how satisfied they were with this support.

In addition to measuring actual (i.e. objective) social isolation in adults with hearing loss, it is also vital to measure perceived (i.e. subjective) social isolation in this population. Research suggests that an individual with hearing loss could frequently attend social events and have a large social network (i.e. low actual social isolation) and yet still experience feelings of loneliness and exclusion, such as feeling left out during group discussions (i.e. high perceived social isolation) (Heffernan et al. 2016; Vas et al. 2017). Conversely, there are also likely to be many adults with hearing loss who have a small number of social connections and social activities (i.e. high actual social isolation) but who are satisfied with this degree of participation (i.e. low perceived social isolation). Research from other healthcare fields has confirmed that actual and perceived social isolation are not necessarily correlated; thus highlighting the importance of measuring both constructs. (Cornwell and Waite 2009b; Holt-Lunstad et al. 2010; Coyle and Dugan 2012). Furthermore, in terms of their impact on wellbeing, the quality of one's social connections may be more important than their quantity (Pinquart and Sörensen 2000).

A number of studies have measured loneliness in adults with hearing loss, which is arguably the equivalent of perceived social isolation (Gierveld and Tilburg 2006; Hawthorne 2008). For example, Dawes et al. (2015), assessed social isolation in adults with hearing loss using a single item, which asked whether respondents regularly feel lonely. However, single item measures tend to have poor reliability, validity, and responsiveness (Hobart et al. 2007; Dawes et al. 2015). Several studies used the 11-item Loneliness Scale (de Jong-Gierveld and Kamphuls 
1985) to assess emotional loneliness (i.e. the feeling of lacking an intimate relationship) and social loneliness (i.e. the feeling of lacking a broader social network) in adults with hearing loss (e.g. Kramer et al. 2002; Weinstein et al. 2016). An abbreviated 6-item version of this scale is now available (Gierveld and Tilburg 2006). However, this measure, like many of the brief perceived social isolation measures utilized in hearing research, was designed for the general population. The disadvantage of such generic measures is that they tend to lack sensitivity to clinically meaningful improvements when compared to hearing-specific measures (Bess 2000). Therefore, a hearing-specific measure of perceived social isolation that is brief yet valid is needed.

To this end, we developed the Social Isolation Measure (SIM). This 5-item questionnaire was derived from the Social Perceptions subscale of the Social Participation Restrictions Questionnaire (SPaRQ) (Heffernan et al. 2018a; Heffernan et al. 2018b). The SPaRQ is a 19item, self-administered, hearing-specific outcome measure. It contains a 9-item Social Behaviours subscale, which measures difficulty performing actions in a social context due to hearing loss (e.g. difficulty persevering with lengthy conversations), and a 10-item Social Perceptions subscale, which measures the impact of hearing loss on thoughts and feelings experienced in a social context (e.g. feeling isolated during group conversations). It was given an 11-point response scale because a relatively large number of response categories are associated with greater responsiveness, reliability, and validity (Alwin 1997; Weng 2004; Leung 2011). The first iteration of the SPaRQ was generated through a literature review and individual, semistructured interviews with adults with hearing loss, clinicians, and academics (Heffernan et al. 2016). The content of the SPaRQ was then evaluated and refined through cognitive interviews with adults with hearing loss and a survey of clinicians and academics (Heffernan et al. 2018a). 
The SPaRQ was further evaluated and refined in a quantitative study using modern and traditional psychometric analysis techniques, including Rasch analysis (Heffernan et al. 2018b). Each subscale was found to have strong measurement properties (e.g. internal consistency, construct validity). Therefore, the SPaRQ is suitable for use in either research or clinical practice to measure the impact of auditory rehabilitation interventions on social participation in adults with hearing loss. It is particularly suitable for clinical trials, where high-quality, standardized tools are required.

The SIM was developed to provide a means of rapidly assessing the impact of auditory rehabilitation interventions on perceived social isolation in adults with hearing loss. An abbreviated measure would be particularly useful in clinical practice, where there is often limited time for outcome measurement. It would also be suitable for use in research, such as in trials where numerous outcomes must be assessed without placing undue burden on participants. Whilst the measurement properties of the SPaRQ have already been established, the properties of its abbreviated version, the SIM, have not yet been investigated. It is important to assess the properties of a questionnaire anew each time that it is altered, as changes to format and content can lead to changes in these properties (Hyde 2000). Therefore, the aim of this research was to evaluate the measurement properties of the SIM, including its reliability, validity, and interpretability.

\section{Materials and methods}

\section{Design}

This was a quantitative study consisting of two phases. Phase 1 was designed to assess the construct validity, internal consistency, and interpretability of the SIM. It entailed asking adults 
with hearing loss to complete the SIM and several related questionnaires. Phase 2 sought to assess the test-retest reliability of the SIM. In this Phase. the same sample of participants were required to complete the SIM again 2-3 weeks following Phase 1. An interval of 2-4 weeks is typically long enough to prevent participants from recalling their previous responses, and short enough to ensure that a clinical change that would affect reproducibility has not occurred (Scholtes et al. 2011). The study was approved by the Faculty of Medicine and Health Sciences Research Ethics Committee, University of Nottingham, UK.

\section{Recruitment and sampling}

In Phase 1, a minimum of 100 participants was required to assess construct validity and internal consistency. In Phase 2, a minimum of 50 participants was required to assess test-retest reliability (Terwee et al. 2007; Hobart et al. 2012). The inclusion criteria were self-reported: (1) hearing loss, (2) minimum age of 18 years, (3) good written English language ability, and (4) normal or corrected-to-normal vision. The exclusion criteria were self-reported: (1) cognitive decline or dementia that would necessitate assistance in completing a questionnaire and (2) profound hearing loss. Furthermore, to accurately assess test-retest reliability, the participants were required to have a stable hearing loss between Phase 1 and Phase 2.

The majority of participants (91.4\%) were recruited via an invitation email sent to the National Institute for Health Research (NIHR) Nottingham Biomedical Research Centre (BRC) participant database. This database contains the contact details of adults who have consented to being contacted about participating in hearing research. The remaining participants $(8.6 \%)$ were recruited via the 'Deafness and hearing loss' online forum on the website of Action on Hearing Loss: a UK-based charity.

\section{Participants}


One-hundred and sixteen adults with hearing loss participated in Phase 1 (see Table 1). Ninetyfive of these participants also completed Phase 2. Twenty-nine participants were removed from the Phase 2 data analysis because their scores could have impeded the evaluation of test-retest reliability. Firstly, 15 participants were removed because they reported that their hearing had changed since Phase 1 (e.g. middle ear infection, sound processor adjustment). Secondly, 14 participants were removed because they completed Phase 2 outside of the specified 2-3 week interval following Phase 1. Therefore, 66 participants were included in the Phase 2 data analysis (see Table 1).

\section{Materials}

In Phase 1, participants provided demographic information (e.g. gender, age, hearing aid use) and completed the 5-item Davis et al. (2007) hearing loss screening questionnaire. They also completed the SIM and three additional questionnaires in order to provide a means of assessing the measurement properties of the SIM. The additional instruments were selected because they measure constructs that are related to perceived social isolation and there is published evidence to support their measurement properties. The questionnaires used are described below.

\section{Social Isolation Measure (SIM)}

This 5-item questionnaire is a standardized, self-administered, hearing-specific, patient-reported outcome measure that was designed to assess the perceived social impact of hearing loss (see Table 2). Respondents rate whether they agree with the statements in the items using an 11-point response scale with labelled endpoints ("Completely disagree"=0, "Completely agree"=10). A total score is obtained by summing the scores for each item, with the maximum possible total score being 50. Participants who normally wore a hearing aid(s) were asked to answer the items based on wearing their aid(s). 


\section{Hearing Handicap Inventory for the Elderly (HHIE)}

This 25 -item questionnaire is a hearing-specific measure of the social/situational and emotional impact of hearing loss (Ventry and Weinstein 1982). The items are accompanied by a 3-point response scale ("Yes", "Sometimes", and "No"). The maximum possible total score is 100. Scores of 16 or less are indicative of 'no handicap', scores in the range of 17-42 are indicative of 'mildmoderate handicap', and scores of 43 or greater are indicative of 'significant handicap' (Weinstein and Ventry 1983). Participants can be instructed to answer the HHIE based either on aided or unaided hearing, depending on the requirements of individual investigators (Ventry and Weinstein 1982). In this study, participants who use a hearing aid(s) were instructed to answer based on wearing their aid(s). This aligned the instructions of the HHIE and the SIM, which was considered to be appropriate for the construct validity analysis and less confusing for the participants.

Shortened World Health Organization Disability Assessment Schedule 2.0 (WHODAS2.0)

This 12-item questionnaire is a generic measure of six activity and participation domains: understanding and communication, mobility, self-care, getting along with others, life activities, and societal participation (Üstün et al. 2010). Participants rate how much difficulty they have experienced in these domains across the past 30 days using a 5-point scale ("None", "Mild", "Moderate", "Severe", and "Extreme or cannot do").

University of California, Los Angeles (UCLA) Loneliness Scale-Version 3 (V3)

This 20-item questionnaire is a generic measure of loneliness (Russell 1996). Participants rate how often they have experienced each item statement using a 4-point scale ("Never", "Rarely", 
"Sometimes", and "Often"). These include statements about having companionship, having social support, and feeling close to others.

In Phase 2, participants completed the SIM again and reported whether there had been any change to their hearing since Phase 1 using a 3-point scale ("No change", "Some change" or "Substantial change"). They described any change in an open-text box.

\section{Procedure}

Potential participants who expressed an interest in the study were provided with a study information sheet and given the opportunity to contact the research team via email or telephone with any queries. In Phase 1, those who wished to participate were sent an email containing a link that enabled them to access an online survey. This survey was created and delivered using the Bristol Online Survey (BOS) tool (www.onlinesurveys.ac.uk/). Participants were asked to complete this survey at their own pace from home or a location of their choice. The survey contained a consent form, alongside the demographics and hearing loss screening questions. It also contained the SIM, HHIE, shortened WHODAS2.0, and UCLA Loneliness Scale-V3. Once participants had submitted their Phase 1 responses via the BOS tool, they were no longer able to access these responses. Phase 2 occurred 2-3 weeks following Phase 1. Participants were sent an email containing a link that provided them with access to another online survey. This survey requested that they complete the SIM for a second time and report any changes to their hearing since Phase 1 . Throughout the study, participants had the opportunity to contact the researchers via telephone or email with any queries. All participants were entered into a prize draw for a chance to win one of three gift vouchers worth $£ 75$ GBP, $£ 50$ GBP, and $£ 25$ GBP.

\section{Data analysis}


A summary of the statistical analyses conducted is available as Supplemental Material 1 (http://tandfonline.com/doi/suppl).

\section{Construct validity}

This is the extent to which the scores of a test correspond with hypotheses based on the assumption that the test is a valid measure of the target construct. This includes predictions about the relationship between the test and other measures. At least $75 \%$ of these predictions should be confirmed (Terwee et al. 2007). In this study, Spearman's rank order correlation coefficient $\left(\mathrm{r}_{\mathrm{s}}\right)$ was used to assess predictions about the relationship between the SIM and three additional instruments. Specifically, it was predicted that the SIM would have a strong, positive correlation ( \pm 0.6 or above) with a related hearing-specific measure (i.e. HHIE) and moderate, positive correlations $( \pm 0.3$ to \pm 0.59$)$ with two related generic measures (i.e. shortened WHODAS2.0 and UCLA Loneliness Scale-V3).

\section{Internal consistency}

This is the degree to which items in a test are inter-related; indicating that they measure the same construct (Terwee et al. 2007). In this study, Cronbach's alpha was used to assess this property. This statistic should fall within the range of 0.7-0.95 (Terwee et al. 2007; De Vet et al. 2011). Additional indicators of internal consistency used in this study were the mean corrected interitem correlation, which should fall within the range of $0.3-0.7$, and the mean corrected item-total correlation, which should be $\geq 0.3$ (Ferketich 1991; Kline 2013).

\section{Interpretability}

This is the extent to which qualitative meaning can be attributed to the quantitative scores of a test (Terwee et al. 2007). Receiver Operating Characteristic (ROC) analyses were used to assess 
this property. Specifically, ROC analyses assessed the ability of the SIM to accurately distinguish between adults with hearing loss who belong to adjacent categories of hearing difficulty (Greiner et al. 2000; Fackrell et al. 2018). Hearing difficulty was measured by the HHIE. Firstly, the SIM was assessed in terms of its capacity to accurately differentiate between participants with no hearing difficulty (HHIE score $\leq 16)$ and participants with mild-moderate hearing difficulty (HHIE score $=17-42$ ). Secondly, the SIM was assessed in terms of its capacity to accurately differentiate between participants with mild-moderate hearing difficulty (HHIE score $=17-42$ ) and participants with significant hearing difficulty (HHIE score $\geq 43)$. ROC curves plotted sensitivity on the y-axis and 1 - specificity on the x-axis. The Area Under the ROC Curve (AUC) provided a global summary statistic representing the ability of the SIM to accurately discriminate between participants in different hearing difficulty categories (Greiner et al. 2000). An AUC of 0.5 means that there is a $50 \%$ probability that the test cannot differentiate between two adjacent categories of patients. Therefore, AUC values of $\geq 0.7$ are desirable (Eng 2005; Fackrell et al. 2018). ROC analyses were also used to identify SIM cut-off scores for each category that had the optimal balance of sensitivity and specificity (Brennan et al. 2016).

\section{Test-retest reliability}

Reliability is the extent to which the test is free from measurement error. Test-retest reliability specifically refers to the extent to which scores for stable participants are the same for repeated measurements across time (Terwee et al. 2007; Scholtes et al. 2011). The test-retest reliability of the Phase 1 and Phase 2 SIM scores was assessed via a two-way random, single measures Intraclass Correlation Coefficient (ICC) for agreement. An ICC of $\geq 0.70$ is required (Terwee et al. 2007). 
Heffernan

\section{Results}

Descriptive statistics and Cronbach's alpha values for each measure are available as Supplemental Material 2 (http://tandfonline.com/doi/suppl).

\section{Construct validity}

There was a strong, positive correlation between the SIM and the HHIE $\left(r_{s}=0.761, p<0.001\right)$, a moderate, positive correlation between the SIM and the shortened WHODAS2.0 $\left(r_{s}=0.377\right.$, $p<0.001$ ), and a moderate, positive correlation between the SIM and the UCLA Loneliness Scale-V3 $\left(r_{s}=0.426, p<0.001\right)$.

\section{Internal consistency}

The Cronbach's alpha for the SIM was 0.943 . The mean corrected inter-item correlation was 0.772. The mean corrected item-total correlation was 0.847 .

\section{Interpretability}

The first ROC analysis (see Figure 1) assessed the ability of the Phase 1 SIM total score to accurately identify 49 participants in the 'mild-moderate hearing difficulty' category from a sample that also contained 16 participants in the 'no hearing difficulty' category. The AUC was $0.899(95 \% \mathrm{CI}=0.812-0.986, p<0.001)$. A SIM cut-score of 18.50 gave the best accuracy for the distinction between these two categories (sensitivity $84 \%$, specificity $87 \%$ ).

The second ROC analysis assessed the ability of the Phase 1 SIM total score to accurately identify 52 participants in the 'significant hearing difficulty' category from a sample that also contained 49 participants in the 'mild-moderate hearing difficulty' category. The AUC was 0.811 
Heffernan

$(95 \% \mathrm{CI}=0.725-0.898, p<0.001)$. A SIM cut-score of 33.50 gave the best accuracy for the distinction between these two categories (sensitivity $79 \%$, specificity $80 \%$ ).

\section{Test-retest reliability}

The Phase 1 and Phase 2 SIM scores had an ICC of $0.77(95 \%$ CI=0.649-0.853, F(65)=7.99, $p<0.001)$.

\section{Discussion}

This research aimed to evaluate the measurement properties of the hearing-specific Social Isolation Measure (SIM) in order to determine whether it is suited for use in research and practice. The results demonstrate that the SIM has strong measurement properties in accordance with published standards (Terwee et al. 2007). Firstly, as predicted, the SIM displayed a strong association with a related, hearing-specific measure (i.e. HHIE) and moderate associations with related, generic measures (i.e. shortened WHODAS2.0, UCLA Loneliness Scale-V3). This provides support for the construct validity of the SIM. Secondly, the SIM was found to have high internal consistency, suggesting that its items are likely to measure the same construct. However, the mean corrected inter-item correlation (0.772) was just outside of the required range $(0.3-0.7)$. A high degree of internal consistency can be an indication that the questionnaire contains redundant items (Pesudovs et al. 2007). However, a previous study used Rasch analysis to demonstrate that the items in the SPaRQ, from which the SIM is derived, are free of response dependency (i.e. redundancy) (Heffernan et al. 2018b). The high internal consistency could instead be due to the relatively large number of categories in the response scale (Weng 2004). Thirdly, the ROC analyses demonstrated that the SIM has the capacity to accurately discriminate between participants who belong to different categories of hearing difficulty. This supports the interpretability of the SIM, which is the degree to which qualitative meaning can be given to its 
quantitative scores (Terwee et al. 2007). Finally, the SIM displayed strong test-retest reliability, denoting that repeated measurements in participants with stable hearing will produce similar responses (Terwee et al. 2007). Therefore, the SIM is suitable for use in research and practice as a measure of perceived social isolation in adults with hearing loss.

One of the main advantages of the SIM is that, in addition to having strong reliability and validity, it places minimal burden on respondents, clinicians, and researchers. Specifically, it contains just five items that adults with hearing loss can self-administer. These items were originally developed through qualitative research with adults with hearing loss and have been shown to be clear and easy to complete (Heffernan et al. 2016; Heffernan et al. 2018a). Furthermore, scoring the SIM entails a straight-forward summation of the raw scores for each item. Therefore, this measure is suitable for use in clinical practice as a rapid means of measuring an important patient-reported outcome. The brevity and simplicity of the SIM are crucial because the time pressure associated with lengthy or complex measures is a major impediment to outcome measurement in clinical practice (Dillon and So 2000; Duncan and Murray 2012). Moreover, the feasibility and burden of a measure are now recognized as being of equal importance to the more traditional measurement properties of reliability and validity (Reeve et al. 2013; Prinsen et al. 2016). The SIM is also suitable for use in research, particularly studies in which respondent burden must be minimized, such as when the participants are frail or when numerous outcomes must be assessed (Reeve et al. 2013; Prinsen et al. 2016).

Social isolation in adults with hearing loss is an increasingly important area of research. Several studies have found that hearing loss is associated with depression, cognitive decline, and dementia (Gopinath et al. 2009; Nachtegaal et al. 2009; Lin et al. 2011; Keidser et al. 2015; Livingston et al. 2017). It has been proposed that hearing loss leads to social isolation, which 
could then contribute to the development of depressive and cognitive symptoms (Lin and Albert 2014; West 2017). However, research to date indicates that the relationships between these factors are likely to be complex and potentially multi-directional (Andrade et al. 2017). For instance, some studies have indicated that there is an independent relationship between hearing loss and depression that is not greatly mediated by social isolation (Hawthorne 2008; Keidser and Seeto 2017). Another study found that depression mediated the relationship between hearing loss and social participation (Andrade et al. 2017). Furthermore, the relationships between these factors could differ depending on whether actual or perceived social isolation has been measured (Holt-Lunstad et al. 2010; Keidser and Seeto 2017). For example, a study of the general population found that feelings of loneliness, rather than actual social isolation, predicted dementia onset (Holwerda et al. 2012). Therefore, additional research is required on the links between hearing loss, other health conditions, and the two forms of social isolation.

Research is also required to identify optimal interventions for social isolation in adults with hearing loss. A recent Cochrane review demonstrated that hearing aids can improve social participation in this population (Ferguson et al. 2017). Nevertheless, many individuals with hearing loss do not use or under-use their hearing aids (McCormack and Fortnum 2013). One reason for low adherence to hearing aids is that they are not necessarily beneficial in social situations, particularly group conversations and conversations in background noise (McCormack and Fortnum 2013; Heffernan et al. 2016). Auditory rehabilitation programs could provide an alternative means of tackling social isolation in adults with hearing loss (Hawkins 2005; Thorén et al. 2014). These include online and face-to-face programs, as well as individual and group programs. They typically provide counselling and education to increase communication strategy use, hearing aid use, social participation, and quality of life (Hawkins 2005; Thorén et al. 2014). 
In addition, social isolation interventions from other healthcare fields could be adapted for adults with hearing loss. These interventions include home visiting, individual or group counselling, cognitive behavioural therapy (CBT), support groups, computer and internet training, social activities, and physical activities (Dickens et al. 2011; Masi et al. 2011). These interventions should have a theoretical foundation and provide patients with opportunities for input or control (Dickens et al. 2011). The optimal interventions for perceived social isolation are those that address maladaptive cognition, such as CBT (Masi et al. 2011).

\section{Limitations}

The study sample may not have been representative of all adults with hearing loss. The majority of participants came from the NIHR Nottingham BRC participant database, which contains many experienced hearing aid users and experienced research participants. Furthermore, as this was an online study, it was not possible to obtain actual hearing thresholds for the participants. Future research should examine the relationship between the SIM and pure-tone audiometry. Another limitation is that the SIM was administered to participants alongside the three questionnaires used for assessing construct validity. Ideally, the questionnaire undergoing validation should be administered on a separate occasion to the other questionnaires so that their responses do not influence one another (De Vet et al. 2011). However, it is common practice in studies assessing construct validity to administer all questionnaires on a single occasion (Chisolm et al. 2005; Post et al. 2008). Furthermore, the ROC analysis presented in this study had some limitations. Firstly, it was not possible to assess the ability of the SIM to accurately differentiate between participants with different levels of hearing-specific perceived social isolation because no goldstandard measure of this construct was available. Therefore, the ability of the SIM to distinguish between participants with different levels of hearing difficulty, as measured by the HHIE, was 
assessed. Secondly, there was a relatively small number of participants $(n=16)$ in the "no hearing difficulty' category, which could have affected the ROC analysis results relating to that group. A potential limitation of the SIM itself is that it has just five items, which could affect its content validity, or the degree to which the target construct has been comprehensively captured by the questionnaire (Terwee et al. 2007). Longer questionnaires that assess social isolation are available for contexts in which content validity is of particular importance. However, it is valuable to have a brief, hearing-specific tool for circumstances wherein time management, feasibility, and minimizing patient and administrator burden are priorities.

\section{Conclusions}

Social isolation is an increasingly important issue in both research and practice in audiology. It is recognized as being one of the major negative consequences of hearing loss and has been linked to other serious health conditions, including depression and dementia. This research has produced a new hearing-specific, perceived social isolation questionnaire that displays a range of strong psychometric properties, including construct validity, internal consistency, and test-retest reliability. This measure could be used in clinical practice and research when a brief yet highquality measure is required to rapidly assess social isolation in adults with hearing loss. Future research should evaluate the responsiveness of this measure. 


\section{Acknowledgements}

The authors would like to thank Kathryn Fackrell, David Maidment, and Sandra Smith of the NIHR Nottingham BRC, the Patient and Public Involvement representative and the participants for their assistance with this study.

Please contact the NIHR Nottingham BRC via hearingnihr@nottingham.ac.uk to obtain a copy of the Social Isolation Measure.

\section{Declaration of interest}

This paper presents independent research funded by the NIHR Biomedical Research Centre Programme and the 2017 Action on Hearing Loss (AOHL) Summer Studentship scheme. The views expressed are those of the authors and not necessarily those of the NHS, the NIHR, AOHL, or the UK Department of Health and Social Care. 
Table 1. Demographic Information of the Participants

\begin{tabular}{cc|c|c}
\hline \multicolumn{2}{c|}{ Demographic } & Phase 1 & Phase 2 \\
\hline \multirow{2}{*}{ Gender } & Male & 61 & 33 \\
& Female & 55 & 33 \\
\hline \multirow{3}{*}{ Age (years) } & Mean & 63.04 & 62.48 \\
& SD & 12.09 & 11.17 \\
& Range & $21-94$ & $23-84$ \\
\hline Estimated & Mean & 16.96 & 16.70 \\
duration of & SD & 16.56 & 16.14 \\
hearing loss & Range & $1-72$ & $1-72$ \\
(years) & Never & 11 & 6 \\
\hline \multirow{3}{*}{ Hearing aid use } & Sometimes & 33 & 16 \\
& Always & 72 & 44 \\
\hline
\end{tabular}


Table 2. Summary of the Content of the Social Isolation Measure

\begin{tabular}{c|l}
\hline Item & Abbreviated item content \\
\hline 1 & Isolated during group conversations \\
\hline 2 & Find social gatherings stressful \\
\hline 3 & Frustrated by being left out \\
\hline 4 & Isolated at get-togethers with family and friends \\
\hline 5 & Unenthusiastic about joining in conversations \\
\hline
\end{tabular}


Figure 1. Receiver operating characteristic curves for identifying optimal cut-scores for the social isolation measure
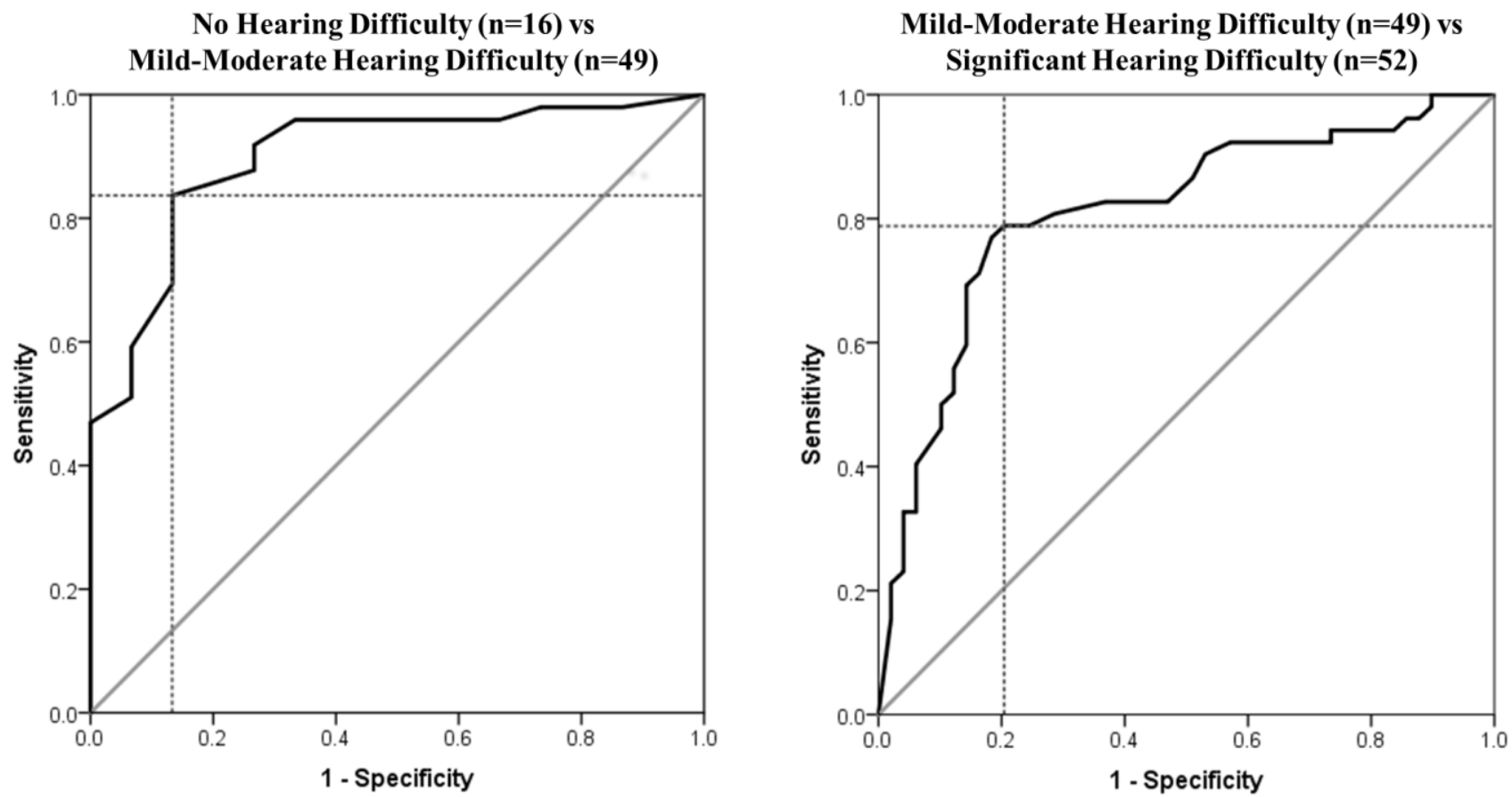

Sensitivity is plotted on the $\mathrm{y}$-axis and 1 - specificity is plotted on the $\mathrm{x}$-axis. The solid black line outlines the AUC. The solid grey line denotes $50 \%$ probability of accurately classifying hearing difficulty. The intersection of the broken grey lines displays the cut-score that provides an optimal balance of sensitivity and specificity. 


\section{References}

Alwin, D. F. (1997). Feeling thermometers versus 7-point scales: which are better? Sociological Methods and Research, 25(3): 318-340. doi:10.1177/0049124197025003003.

Andrade, C. C., Pereira, C. R., and Da Silva, P. A. (2017). The silent impact of hearing loss: using longitudinal data to explore the effects on depression and social activity restriction among older people. Ageing and Society, 1-22.

Bess, F. H. (2000). The role of generic health-related quality of life measures in establishing audiological rehabilitation outcomes. Ear and Hearing, 21(4): 74S-79S.

Boothroyd, A. (2007). Adult aural rehabilitation: what is it and does it work? Trends in Amplification, 11(2): 63-71.

Brennan, L., Siderowf, A., Rubright, J. D., Rick, J., Dahodwala, N., Duda, J. E., Hurtig, H., Stern, M., Xie, S.X., Rennert, L. and Karlawish, J. (2016). The penn parkinson's daily activities questionnaire-15: psychometric properties of a brief assessment of cognitive instrumental activities of daily living in parkinson's disease. Parkinsonism and Related Disorders, 25: 21-26. doi:https://doi.org/10.1016/j.parkreldis.2016.02.020

Chisolm, T. H., Abrams, H. B., McArdle, R., Wilson, R. H., and Doyle, P. J. (2005). The WHODAS II: Psychometric properties in the measurement of functional health status in adults with acquired hearing loss. Trends in Amplification, 9(3): 111-126.

Cornwell, E. Y., and Waite, L. J. (2009a). Measuring social isolation among older adults using multiple indicators from the nshap study. The Journals of Gerontology: Series B, 64B(S1): i38-i46. doi:10.1093/geronb/gbp037

Cornwell, E. Y., and Waite, L. J. (2009b). Social disconnectedness, perceived isolation, and health among older adults. Journal of Health and Social Behavior, 50(1): 31-48. doi:10.1177/002214650905000103

Coyle, C. E., and Dugan, E. (2012). Social isolation, loneliness and health among older adults. Journal of Aging and Health, 24(8): 1346-1363. doi:10.1177/0898264312460275

Davis, A., Smith, P., Ferguson, M., Stephens, D., \& Gianopoulos, I. (2007). Acceptability, benefit and costs of early screening for hearing disability: A study of potential screening tests and models. Health Technology Assessment, 11(42): 1-294.

Dawes, P., Emsley, R., Cruickshanks, K.J., Moore, D.R., Fortnum, H., Edmondson-Jones, M., McCormack, A. and Munro, K.J. (2015). Hearing loss and cognition: the role of hearing aids, social isolation and depression. PLoS One, $10(3$ (e0119616)): 1-9. doi:10.1371/journal.pone.0119616

de Jong-Gierveld, J., and Kamphuls, F. (1985). The development of a Rasch-type loneliness scale. Applied Psychological Measurement, 9(3): 289-299. 
De Vet, H. C., Terwee, C. B., Mokkink, L. B., and Knol, D. L. (2011). Measurement in medicine: a practical guide. Cambridge University Press.

Dickens, P.A., Richards, S.H., Greaves, C.J. and Campbell, J.L. (2011). Interventions targeting social isolation in older people: a systematic review, BMC Public Health, 11: 647.

Dillon, H., and So, M. (2000). Incentives and obstacles to the routine use of outcomes measures by clinicians. Ear and hearing, 21(4): 2S-6S.

Duncan, E. A., and Murray, J. (2012). The barriers and facilitators to routine outcome measurement by allied health professionals in practice: a systematic review. BMC Health Services Research, 12(1): 96.

Eng, J. (2005). Receiver operating characteristic analysis: a primer. Academic Radiology, 12(7): 909-916. doi:10.1016/j.acra.2005.04.005

Fackrell, K., Hall, D. A., Barry, J. G., and Hoare, D. J. (2018). Performance of the Tinnitus Functional Index as a diagnostic instrument in a UK clinical population. Hearing Research, 358: 74-85. doi:https://doi.org/10.1016/j.heares.2017.10.016

Ferguson, M. A., Kitterick, P. T., Chong, L. Y., Edmondson-Jones, M., Barker, F., and Hoare, D. J. (2017). Hearing aids for mild to moderate hearing loss in adults. Cochrane Database of Systematic Reviews(9). doi:10.1002/14651858.CD012023.pub2

Ferketich, S. (1991). Focus on psychometrics. Aspects of item analysis. Research in Nursing and Health, 14(2): 165-168.

Garland, A. F., Kruse, M., and Aarons, G. A. (2003). Clinicians and outcome measurement: What's the use? The Journal of Behavioral Health Services and Research, 30(4): 393405. doi:10.1007/bf02287427

Gierveld, J. D. J., and Tilburg, T. V. (2006). A 6-item scale for overall, emotional, and social loneliness: confirmatory tests on survey data. Research on Aging, 28(5): 582-598. doi:10.1177/0164027506289723

Glass, T. A., De Leon, C. F. M., Bassuk, S. S., and Berkman, L. F. (2006). Social engagement and depressive symptoms in late life longitudinal findings. Journal of Ageing and Health, 18(4): 604-628.

Gopinath, B., Wang, J.J., Schneider, J., Burlutsky, G., Snowdon, J., McMahon, C.M., Leeder, S.R. and Mitchell, P. (2009). Depressive symptoms in older adults with hearing impairments: the blue mountains study. Journal of the American Geriatrics Society, 57(7): 1306-1308. doi:10.1111/j.1532-5415.2009.02317.x

Greiner, M., Pfeiffer, D., and Smith, R. D. (2000). Principles and practical application of the receiver-operating characteristic analysis for diagnostic tests. Preventive Veterinary Medicine, 45(1), 23-41. doi:https://doi.org/10.1016/S0167-5877(00)00115-X 
Hawkins, D. B. (2005). Effectiveness of Counseling-Based Adult Group Aural Rehabilitation Programs: A Systematic Review of the Evidence, Journal of the American Academy of Audiology, 16: 485-93.

Hawthorne, G. (2006). Measuring Social isolation in older adults: development and initial validation of the friendship scale. Social Indicators Research, 77(3): 521-548. doi:10.1007/s11205-005-7746-y

Hawthorne, G. (2008). Perceived social isolation in a community sample: its prevalence and correlates with aspects of peoples' lives. Social Psychiatry and Psychiatric Epidemiology, 43(2): 140-150. doi:10.1007/s00127-007-0279-8

Heffernan, E., Coulson, N., and Ferguson, M. (2018). Development of the Social Participation Restrictions Questionnaire (SPaRQ) through consultation with adults with hearing loss, researchers, and clinicians: A content evaluation study. International Journal Audiology. doi:10.1080/14992027.2018.1483585

Heffernan, E., Coulson, N. S., Henshaw, H., Barry, J. G., and Ferguson, M. A. (2016). Understanding the psychosocial experiences of adults with mild-moderate hearing loss: An application of Leventhal's self-regulatory model. International Journal Audiology, 55(S3): S3-S12. doi:10.3109/14992027.2015.1117663

Heffernan, E., Maidment, D., Barry, J., and Ferguson, M. (2018). Refinement and validation of the social participation restrictions questionnaire: an application of rasch analysis and traditional psychometric analysis techniques. Ear and Hearing. doi:10.1097/AUD.0000000000000618

Hobart, J. C., Cano, S. J., Warner, T. T., and Thompson, A. J. (2012). What sample sizes for reliability and validity studies in neurology? Journal of Neurology, 259(12): 2681-2694. doi:10.1007/s00415-012-6570-y

Hobart, J. C., Cano, S. J., Zajicek, J. P., and Thompson, A. J. (2007). Rating scales as outcome measures for clinical trials in neurology: problems, solutions, and recommendations. The Lancet Neurology, 6(12): 1094-1105. doi:http://dx.doi.org/10.1016/S14744422(07)70290-9

Holt-Lunstad, J., Smith, T. B., and Layton, J. B. (2010). Social relationships and mortality risk: a meta-analytic review. PLOS Medicine, 7(7): e1000316. doi:10.1371/journal.pmed.1000316

Holwerda, T. J., Deeg, D. J. H., Beekman, A. T. F., van Tilburg, T. G., Stek, M. L., Jonker, C., and Schoevers, R. A. (2012). Feelings of loneliness, but not social isolation, predict dementia onset: results from the Amsterdam Study of the Elderly (AMSTEL). Journal of Neurology, Neurosurgery and Psychiatry.

Hughes, M. E., Waite, L. J., Hawkley, L. C., and Cacioppo, J. T. (2004). A short scale for measuring loneliness in large surveys: Results from two population-based studies. Research on Aging, 26(6): 655-672. 
Hyde, M. L. (2000). Reasonable psychometric standards for self-report outcome measures in audiological rehabilitation. Ear and Hearing, 21(4), 24S-36S.

Jang, Y., Mortimer, J. A., Haley, W. E., Small, B. J., Chisolm, T. E. H., \& Graves, A. B. (2003). The role of vision and hearing in physical, social, and emotional functioning among older adults. Research on Aging, 25(2): 172-191. doi:10.1177/0164027502250019

Keidser, G., and Seeto, M. (2017). The influence of social interaction and physical health on the association between hearing and depression with age and gender. Trends in Hearing, 21, doi:10.1177/2331216517706395

Keidser, G., Seeto, M., Rudner, M., Hygge, S., and Rönnberg, J. (2015). On the relationship between functional hearing and depression. International Journal Audiology, 54(10), 653-664. doi:10.3109/14992027.2015.1046503

Kline, P. (2013). Handbook of psychological testing. Routledge.

Kramer, S. E., Kapteyn, T. S., Kuik, D. J., and Deeg, D. J. (2002). The association of hearing impairment and chronic diseases with psychosocial health status in older age. Journal of Ageing and Health, 14(1): 122-137.

Krause, N., \& Borawski-Clark, E. (1995). Social class differences in social support among older adults. Gerontologist, 35(4): 498-508.

Leung, S.-O. (2011). A comparison of psychometric properties and normality in 4-, 5-, 6-, and 11-point likert scales. Journal of Social Service Research, 37(4): 412-421. doi:10.1080/01488376.2011.580697

Lin, F. R., and Albert, M. (2014). Hearing loss and dementia - who is listening? Aging and Mental Health, 18(6): 671-673. doi:10.1080/13607863.2014.915924

Lin, F. R., Metter, E. J., O’Brien, R. J., Resnick, S. M., Zonderman, A. B., and Ferrucci, L. (2011). Hearing loss and incident dementia. Archives of Neurology, 68(2): 214-220.

Livingston, G., Sommerlad, A., Orgeta, V., Costafreda, S.G., Huntley, J., Ames, D., Ballard, C., Banerjee, S., Burns, A., Cohen-Mansfield, J. and Cooper, C. (2017). Dementia prevention, intervention, and care. The Lancet, 390(10113): 2673-2734.

Lubben, J. E. (1988). Assessing social networks among elderly populations. Family and Community Health: The Journal of Health Promotion and Maintenance.

Masi, C. M., Chen, H. Y., Hawkley, L. C., \& Cacioppo, J. T. (2011). A meta-analysis of interventions to reduce loneliness. Personality and Social Psychology Review, 15(3): 219-266.

McCormack, A., and Fortnum, H. (2013). Why do people fitted with hearing aids not wear them?, International Journal of Audiology, 52: 360-68. 
Mick, P., and Pichora-Fuller, M. K. (2016). Is hearing loss associated with poorer health in older adults who might benefit from hearing screening? Ear and Hearing, 37(3): e194-e201. doi:10.1097/aud.0000000000000267

Nachtegaal, J., Smit, J. H., Smits, C., Bezemer, P. D., van Beek, J. H., Festen, J. M., and Kramer, S. E. (2009). The association between hearing status and psychosocial health before the age of 70 years: results from an internet-based national survey on hearing. Ear and Hearing, 30(3): 302-312.

National Health and Nutrition Examination Survey Social Support Questionnaire. (2005). Centers for Disease Control and Prevention and National Center for Health Statistics.

NHS England. (2015). Action plan on hearing loss. Department of Health.

NHS England. (2016). Commissioning services for people with hearing loss: a framework for clinical commissioning groups. Department of Health.

Nicholson Jr., N. R. (2009). Social isolation in older adults: an evolutionary concept analysis. Journal of advanced nursing, 65(6): 1342-1352. doi:doi:10.1111/j.13652648.2008.04959.x

Pesudovs, K., Burr, J. M., Harley, C., and Elliott, D. B. (2007). The development, assessment, and selection of questionnaires. Optometry and Vision Science, 84(8): 663-674.

Pinquart, M., and Sörensen, S. (2000). Influences of socioeconomic status, social network, and competence on subjective well-being in later life: A meta-analysis. Psychology and aging, 15(2): 187-224. doi:10.1037/0882-7974.15.2.187

Post, M. W., de Witte, L. P., Reichrath, E., Verdonschot, M. M., Wijlhuizen, G. J., and Perenboom, R. J. (2008). Development and validation of IMPACT-S, an ICF-based questionnaire to measure activities and participation. Journal of Rehabilitation Medicine, 40(8): 620-627.

Prinsen, C.A., Vohra, S., Rose, M.R., Boers, M., Tugwell, P., Clarke, M., Williamson, P.R. and Terwee, C.B. (2016). How to select outcome measurement instruments for outcomes included in a "Core Outcome Set" - a practical guideline. Trials, 17(1), 449. doi:10.1186/s13063-016-1555-2

Reeve, B.B., Wyrwich, K.W., Wu, A.W., Velikova, G., Terwee, C.B., Snyder, C.F., Schwartz, C., Revicki, D.A., Moinpour, C.M., McLeod, L.D. and Lyons, J.C. (2013). ISOQOL recommends minimum standards for patient-reported outcome measures used in patientcentered outcomes and comparative effectiveness research. Quality of Life Research, 22: 1889-1905.

Russell, D. W. (1996). UCLA Loneliness Scale (Version 3): Reliability, validity, and factor structure. Journal of personality assessment, 66(1): 20-40.

doi:10.1207/s15327752jpa6601_2 
Scholtes, V. A., Terwee, C. B., and Poolman, R. W. (2011). What makes a measurement instrument valid and reliable? Injury, 42(3): 236-240. doi:http://dx.doi.org/10.1016/j.injury.2010.11.042

Shankar, A., Hamer, M., McMunn, A., and Steptoe, A. (2013). Social isolation and loneliness: relationships with cognitive function during 4 years of follow-up in the english longitudinal study of ageing. Psychosomatic medicine, 75(2): 161-170. doi:10.1097/PSY.0b013e31827f09cd

Steptoe, A., Shankar, A., Demakakos, P., and Wardle, J. (2013). Social isolation, loneliness, and all-cause mortality in older men and women. Proceedings of the National Academy of Sciences, 110(15): 5797-5801.

Strawbridge, W. J., Wallhagen, M. I., Shema, S. J., and Kaplan, G. A. (2000). Negative Consequences of hearing impairment in old age a longitudinal analysis. Gerontologist, 40(3): 320-326.

Terwee, C.B., Bot, S.D., de Boer, M.R., van der Windt, D.A., Knol, D.L., Dekker, J., Bouter, L.M. and de Vet, H.C. (2007). Quality criteria were proposed for measurement properties of health status questionnaires. Journal of clinical epidemiology, 60(1): 34-42.

Thorén, E.S., Öberg, M., Wänström, G., Andersson, G. and Lunner, T. (2014). A randomized controlled trial evaluating the effects of online rehabilitative intervention for adult hearing-aid users, International Journal of Audiology, 53: 452-61.

Üstün, T. B., Chatterji, S., Kostanjsek, N., Rehm, J., Kennedy, C., Epping-Jordan, J., Saxena, S., von Koff, M. and Pull, C. (2010). Developing the World Health Organization disability assessment schedule 2.0. Bulletin of the World Health Organization, 88(11): 815-823.

Valtorta, N. K., Kanaan, M., Gilbody, S., Ronzi, S., and Hanratty, B. (2016). Loneliness and social isolation as risk factors for coronary heart disease and stroke: systematic review and meta-analysis of longitudinal observational studies. Heart, 102(13): 1009-1016.

Vas, V., Akeroyd, M. A., and Hall, D. A. (2017). A data-driven synthesis of research evidence for domains of hearing loss, as reported by adults with hearing loss and their communication partners. Trends in Hearing, 21, doi:10.1177/2331216517734088

Ventry, I. M., and Weinstein, B. E. (1982). The hearing handicap inventory for the elderly: a new tool. Ear and Hearing, 3(3), 128-134.

Weinstein, B. E., Sirow, L. W., and Moser, S. (2016). Relating hearing aid use to social and emotional loneliness in older adults. American Journal of Audiology, 25(1), 54-61. doi:10.1044/2015_aja-15-0055

Weinstein, B. E., and Ventry, I. M. (1983). Audiometric correlates of the hearing handicap inventory for the elderly. Journal of Speech and Hearing Disorders, 48(4): 379-384. 
Weng, L.-J. (2004). Impact of the number of response categories and anchor labels on coefficient alpha and test-retest reliability. Educational and Psychological Measurement, 64(6): 956972.

West, J. S. (2017). Hearing impairment, social support, and depressive symptoms among U.S. adults: A test of the stress process paradigm. Social Science and Medicine, 192, 94-101. doi:https://doi.org/10.1016/j.socscimed.2017.09.031 


\section{Supplemental Material 1}

Summary of the data analyses conducted in this study.

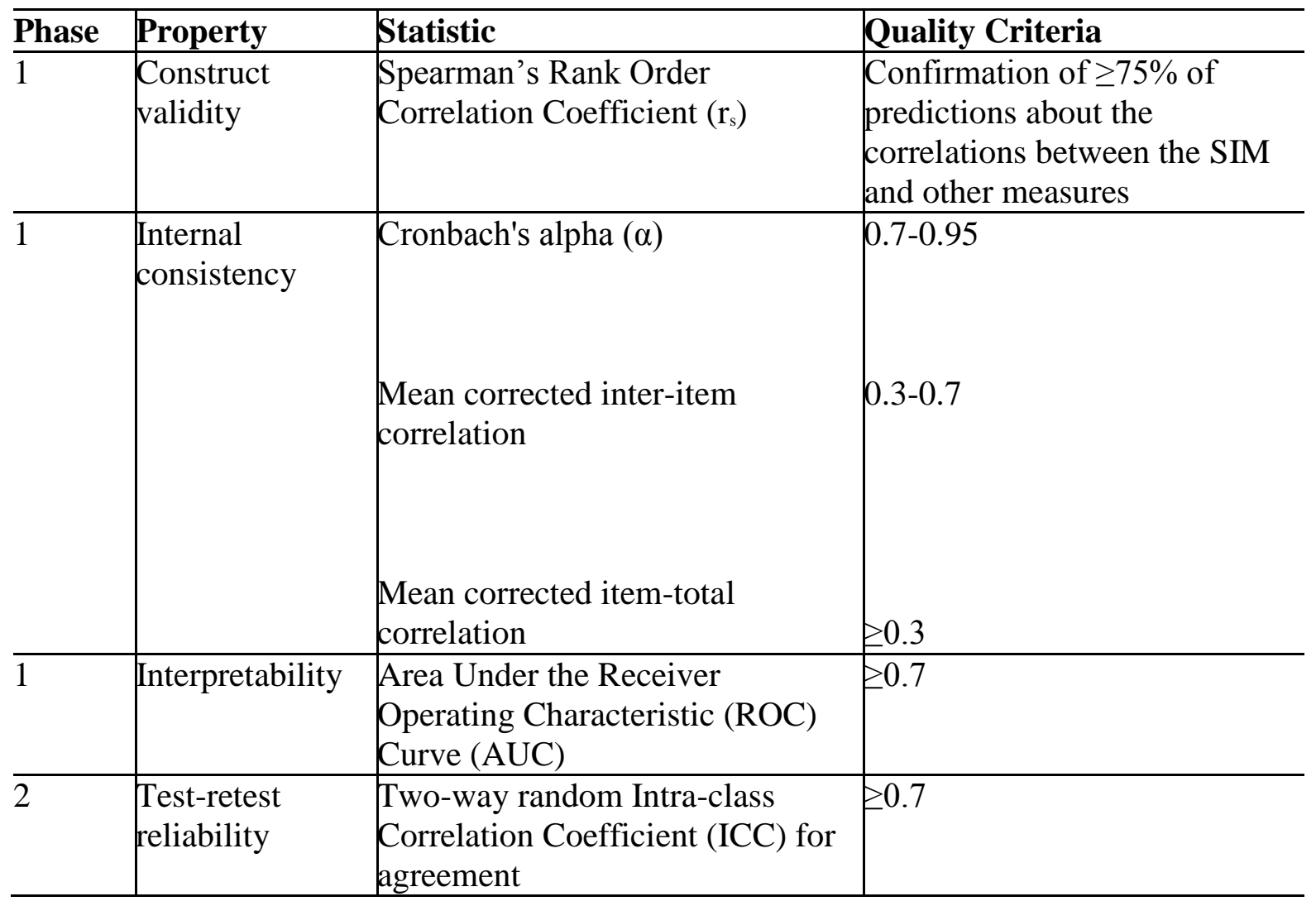




\section{Supplemental Material 2}

Cronbach's alpha $(\alpha)$ values and summary statistics for the total scores of the Phase 1 participants $(\mathrm{N}=116)$ on each of the measures used to assess construct validity.

\begin{tabular}{c|c|c}
\hline Measure & Statistic & Value \\
\hline \multirow{4}{*}{ SIM } & $\alpha$ & 0.943 \\
& Mean & 30.34 \\
& SD & 13.81 \\
& Median & 31 \\
& Range & $0-50$ \\
\hline HHIE & $\alpha$ & 0.933 \\
& Mean & 42.93 \\
& SD & 21.15 \\
& Median & 40 \\
& Range & $6-94$ \\
\hline \multirow{3}{*}{ Shortened WHODAS2.0 } & $\alpha$ & 0.917 \\
& Mean & 7.80 \\
& SD & 8.86 \\
& Median & 5 \\
& Range & $0-40$ \\
\hline UCLA Loneliness Questionnaire & $\alpha$ & 0.955 \\
V3 & Mean & 42.09 \\
& SD & 13.74 \\
& Median & 42 \\
& Range & $20-78$ \\
\hline
\end{tabular}

